\title{
On the condensation of polaritons
}

\author{
Benoit Deveaud-Plédran \\ Ecole Polytechnique Fédérale de Lausanne (EPFL) Station 3, 1015 Lausanne Switzerland \\ (benoit.deveaud-pledran@epfl.ch)
}

Received October 18, 2011; accepted November 23, 2011; posted December 12, 2011 (Doc. ID 156787); published February 1, 2012

\begin{abstract}
Bose-Einstein condensates (BECs), the "fifth state of matter," have in fact been discovered by chance both in liquid helium and in superconductors. Since these early encounters, BECs have been sought for, both in atom vapors and in solids. Here we report on the case of exciton polaritons. We discuss the experimental observation of macroscopically occupied polariton states and their possible attribution to a BEC. We also discuss the possible relation between a polariton condensate, a polariton laser, and a vertical surface-emitting laser (VCSEL). Vortices, superfluidity, Bogoliubov dispersion, half-vortices, and Josephson oscillations are then briefly summarized merrily for sake of discussion of the distinction between a polariton BEC and a VCSEL. (c) 2012 Optical Society of America OCIS codes: $\quad 190.5970,020.1475,320.7130,270.6630$.
\end{abstract}

\section{HISTORICAL SURVEY}

The history of Bose-Einstein condensation started with the seminal papers of Bose [1] and Einstein [2] in 1924 and 1925 , respectively. It had been in fact preceded by the discovery of superconductivity in 1911 [3]; the relationship between the two only appeared much later, after the development of the Bardeen, Cooper, and Schiffer (BCS) theory []. Similarly, helium superfluidity $[\underline{5}, \underline{6}]$ was not initially considered as linked with Bose-Einstein condensate (BEC) and it is only with the far-reaching contributions of London, [7] Landau [8], and Bogoliubov [9] that the clear relation between superfluidity and BECs has been unveiled.

The idea to use excitons in semiconductors for BEC derives from the observation, with BCS, that composite bosons could be very good candidates for condensation, in particular thanks to their mass, which is similar to the mass of an electron. The idea was independently proposed by Moskalenko [10] and Blatt [11], and both papers expose that, being composite bosons with very light mass, excitons should show BEC at reasonable temperatures. Such an idea has been pursued further by Keldysh [12], who described the possible transition from BEC to BCS of excitons.

In the following years, the race for BEC was followed in parallel by the exciton community and the cold atom teams. Claims for condensation have been made by different groups in exciton physics, as early as in the late 1970s $[\underline{13}, 14]$. The different groups have, in particular, been using large bandgap semiconductors, in which the binding energy of excitons is larger and therefore the saturation density would be better suited for condensation. Such claims have, however, subsequently proved to be premature and have not been confirmed yet.

Another idea was subsequently followed in the early 1990s with the proposal to use two coupled quantum wells. There again, a whole series of claims have been made $[15,16,17,18]$ that have not yet been confirmed by the appropriate spatial coherence studies. It is not possible here to summarize all such experiments, and we rather refer the reader to the book edited by Moskalenko and Snoke for a detailed account of the field [19].
Let us just highlight here a few interesting facts. One of the difficulties of experiments on excitons lies in the fact that excitons should undergo a Mott transition [20] from an insulating state (bosonic) to a metallic plasma (fermionic) above some critical density $[21,22]$. Also, it appeared that two-body recombinations (Auger recombination, see, for example, the works of Manzke and coworkers [23], Portnoi et al. [24], and Snoke [25]) between excitons occur as soon as the exciton gas reaches a high density, therefore limiting the maximum achievable density and also giving rise to a large heating the exciton cloud. Last, but not least, excitons in three-dimensional (3D) crystals should be viewed as polaritons (see Hopfield [26]) and, upon cooling of the exciton gas, should transform continuously into photons as there is no energy minimum on the polariton dispersion.

The competing path, atom cooling, being experimentally very challenging, did not progress really until the middle of the 1980s when atom molasses were observed and then cooled down by different techniques, such as evaporative cooling [27]. Such techniques were awarded the Nobel prize in 1995, the year when atom BEC was eventually observed by Cornell, Wieman, and Ketterle [28,29], who were jointly awarded the Nobel prize in 2002. Even if the initial observation consisted basically only in the observation of the condensation of most atoms in the lowest speed state, subsequent measurements, carried out by dozens of teams worldwide, have splendidly confirmed the initial claim, in particular with the demonstration of the long-range order of the condensate $[30,31]$ as well as the clear evidence for its superfluid character [32].

In 1992, in a seminal paper, Weisbuch and coworkers pinned down the appearance of strong coupling between light and excitons in semiconductor microcavities, therefore opening the whole field of microcavity polaritons [33]. As polaritons result form the coupling of two bosonic particles, they inherit this bosonic character. From the photon part, they get a very light mass, and from the excitonic part, they are strongly interacting at high densities. They also carry a spin, common to the exciton as well as to the photon part. Polaritons possess many interesting properties linked with their 
dispersion relation shown in Fig. 1. For our purpose here, the most interesting one is their very light mass.

The BEC phase transition occurs, for bosonic particles, either by increasing the density of particles at a constant temperature or reducing the temperature of an ensemble of particles of given density. In the very simple $3 \mathrm{D}$ idealistic situation, the transition temperature is governed by the wellknown relation:

$$
T_{c}=\frac{2 \pi \hbar^{2}}{m}\left(\frac{n}{2.612 L^{3}}\right)
$$

Below the transition temperature at a given density, or above the transition density at a given temperature, a large fraction of the particles occupies the same quantum state, leading to the formation of long-range spatial coherence with consequences such as superfluidity or superconductivity [34]. The formula easily shows that two parameters directly influence the transition temperature: the mass of the particles and their density. The mass of polaritons being four to five orders of magnitude smaller than that of the electron, one expects a transition temperature that would be easily achievable with standard cryogenic techniques. This idea was initially proposed by Imamoglu et al. [35], who even introduced the acronym BOSER for the idea of stimulated scattering of polaritons leading to the emission of coherent light. As we show in the following, Imamoglu was indeed right and polaritons have shown to be good candidates for a quantum fluid.

One difficulty with excitonic polaritons is that they cannot keep their bosonic character at high densities. Indeed, because of the fermionic components of the exciton, both the exciton has a tendency to bleach at high densities as well as the strong coupling with photons disappear. This has been beautifully demonstrated by Romuald Houdré et al. [36].

Nevertheless, experiments such as the parametric scattering first demonstrated by Savvidis et al. [37] have shown that polaritons do behave as good bosons up to densities large enough to obtain degeneracy and up to quite high temperatures [38]. In short, microcavity polaritons are an ideal candidate for the observation of BEC in solids. It has even been predicted that, by a careful choice of the constituent materials, BEC could be observed up to room temperature in such systems [39].

\section{WHAT IS TO BE MEASURED TO ASSESS BEC}

The large amount of work carried out on the possible observation of BEC of excitons in semiconductors, may it be large gap semiconductors as $\mathrm{CuCl}$ and $\mathrm{Cu}_{2} \mathrm{O}$ or coupled quantum wells, as well as the very lively debate that has taken place around the reported observations has allowed us to discuss beforehand in great detail, the measurements that should be reported to sustain a claim of BEC. This list of observations is not only based on the reported observations in solids, but also on the observations realized in the case of atom vapors as well as on the theoretical work on the subject.

Of course, the observation of a diverging Bose distribution of the particles is a prerequisite to claim for BEC. This is, in fact, the observation that convinced the community in the case of atom vapors when first reported in 1995 [28]. It is important to note that the temperature of the ensemble before the transition was precisely assessed and that the phase transition was observed to occur, at thermal equilibrium, by properly diminishing the temperature. This follows the description given, for example by Pitaevskii and Stringari [34] "This phase transition is associated with the condensation of atoms in the state of lowest energy and is the consequence of quantum statistical effects." In semiconductors, the temperature of excitons is in general not precisely measured, and the thermal equilibrium is usually not clearly assessed.

Beyond the measurement of a proper distribution for the observed species, the transition should be accompanied by an increase of the first-order time coherence of the system, possibly assessed also through a narrowing of the linewidth.

BEC should also consist in a spontaneous symmetry breaking, leading for excitons to a well-defined polarization of the exciton gas. This, however, is difficult to measure as the polarization should be random in the absence of disorder and change for each realization of the condensate. Any averaged measurement, i.e., averaged over a time longer than the first-order coherence time, should lead to an absence of polarization [40].

Second-order coherence is a very important measure, and gives key information about the coherence properties of the ensemble. However, it is clear to theorists that the second order coherence of the system is not a strong enough argument to claim for undisputable BEC.
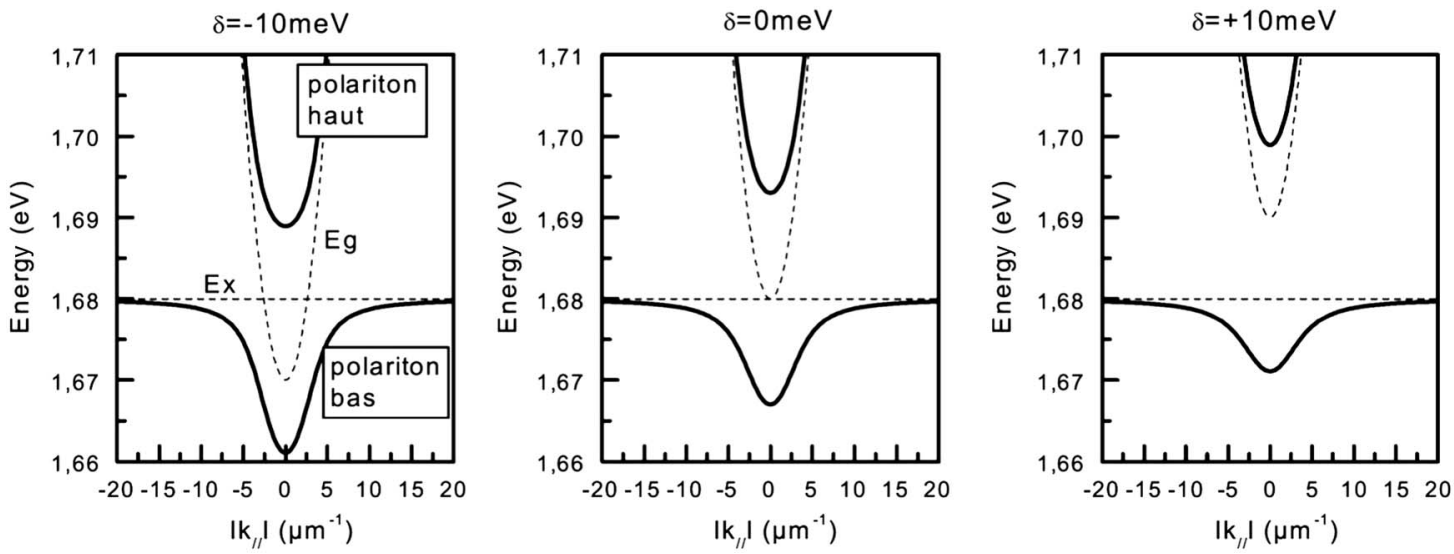

Fig. 1. In-plane dispersion of polaritons for different detunings between the photon mode and the exciton resonance. Left, negative detuning, the photon mode is $10 \mathrm{meV}$ below the exciton resonance; middle, zero detuning; right, the photon mode is 10 meV above the exciton resonance (reproduced from [46] with the agreement of the author). 
Eventually, it has become clear to us that the majority of the theorists, knowledgeable in the field, agree on the fact that the "smoking gun" for demonstration of BEC is the clear evidence for spontaneous build up of long-range spatial coherence of the condensate [41]. Indeed, the idea of long-range coherence and of a macroscopic wave function unifies the understanding of BEC, superfluidity, superconductivity, and lasing. Measurement of the long-range coherence has proven to be a challenging measurement as it requires at the same time interferometric stability and high-quality spatial imaging [31].

\section{WHAT HAS INDEED BEEN OBSERVED}

In a first step, early experiments by Dang et al. [42] and Senellart et al. [43] gave clear evidence for nonlinear emission from nonresonantly excited microcavities. The origin of such nonlinearities has been shown to lie in the stimulated scattering of polaritons in the lower branch. Several experiments, of course including the two cited above, allowed estimating, within a factor of 2 or 3 , the polariton population per state at threshold. In all experiments, the threshold condition does correspond, within experimental uncertainties, to occupations around unity. This is indeed what would be expected for stimulated scattering. The scattering mechanism at threshold is usually polariton-polariton scattering as may be evidenced through the quadratic dependence of the output intensity versus excitation density curve. In some cases, this quadratic part of the dependence is barely observable.

In a second step, polaritons have confirmed to be very good bosons, in particular through the parametric scattering experiments performed first in the group of Baumberg, by Savvidis [35]. Indeed, such experiments, well-known in the field of nonlinear optics, fully rely on the bosonic nature of the excitation of the system. The maximum density of polaritons per state has been observed to be, at low temperature and in the case of CdTe-based microcavities, $10^{5}$ polaritons per state [35]. Parametric amplification of polaritons is now a technique widely used for example to produce high-quality superfluid polariton populations [45].

Polaritons may indeed be considered as good bosons up to densities that approach the saturation density, when the fermionic nature of the excitonic part of the polariton becomes dominant. In simple terms, this saturation density corresponds to the Mott transition in the quantum wells. One of the techniques now unanimously used to help in this matter is to use a large number of quantum wells in the cavity (10 to 12 wells seems to be an optimum). Although the principle may seem simple, the realization appears to be quite challenging as only few sample growers are able to succeed in this task. It is important to give credit to these sample growers who allowed us to perform such interesting experiments: André on CdTe cavities at the University of Grenoble, Oesterle and François Morier-Genoud at EPFL, Lemaitre at LPN Marcoussis, Pfeiffer and West at the University of Princeton, and Worschech at the University of Würzburg to name the key players.

The third step has been to demonstrate that, by choosing proper detuning conditions, a thermal distribution of polaritons, well described by a Boltzmann distribution function, could be obtained at intermediate densities. Evidence for such a thermalization, an amazing observation indeed, as it has to occur within the very short lifetime of the polaritons, of the order of 1 to $10 \mathrm{ps}$ [44].

A fourth step performed in parallel by different groups has been to estimate the density of polariton per state at the threshold for nonlinear emission. The light emission by the polaritons makes it quite easy to estimate the number of polaritons per state and, indeed, this number is, within experimental error, 1 at threshold (a detailed discussion of this point may be found in [46]). This observation, initially made in the case of CdTe cavities, has been confirmed in other material systems (see, for example, [47] or [48]).

At the same time, i.e., when stimulated scattering to the ground state occurs, there is a clear change in the shape of the polariton distribution: it changes from Boltzmann-like to Bose-like. Transition to the Bose distribution has been studied in detail in Kasprzak's PhD thesis [44]. Such changes in the distribution of polaritons have subsequently been reproduced in other configurations.

It has also been checked that, when crossing threshold, the first-order temporal coherence of the polariton cloud abruptly increases at threshold. The value of the temporal coherence observed above this threshold depends on the quality of the exciting laser [49].

Second-order coherence of the condensate has also been measured. The idea was first proposed in a paper by Deng et al. [50], however, their results were not totally convincing and difficult to understand (for better understanding, see the work of Haug [51]). Since then, other measurements have been performed that confirm the transition from a thermal light emission below threshold, to a coherent light at threshold $[\underline{52}, 53]$. The second-order coherence then increases again, as expected theoretically, due to polariton-polariton interactions in the condensate [54].

Last, but not least, the "smoking gun" for Bose condensation, the appearance of long-range order, has been studied using the proper interferometric techniques. We show in Fig. 2 the contrast observed in the interference pattern for a given position on the sample, and for two excitation densities. In the left part of Fig. 2, below the threshold, significant correlations are only observed over a diameter of the order of $2 \times \mu \mathrm{m}$. This is not limited by the resolution of the setup and roughly corresponds to the thermal de Broglie wavelength of the polariton at the temperature of approximately $20 \mathrm{~K}$ as deduced from the observation of the distribution function [55].

With this ensemble of observations, we indeed claimed the observation of BEC of polaritons in 2006 [47]. Long-range order has since then been observed in a number of other systems and in different labs around the world $[\underline{57}, \underline{58}, \underline{59}]$. Polariton condensation has also been claimed, without the demonstration of long-range order, in different systems and in particular in GaN-based cavities at room temperature [60,61].

\section{PHOTON LASING OR POLARITON CONDENSATION?}

The immediate question, which we have been asking ourselves, and that has been raised by a number of colleagues as well during the very lively discussion around our results is the following: Basically, all the observations that have been reported in [55] should be observed in a very similar way in a vertical surface-emitting laser (VCSEL). Why then do we claim polariton BEC and not simply standard VCSEL lasing? Is there 

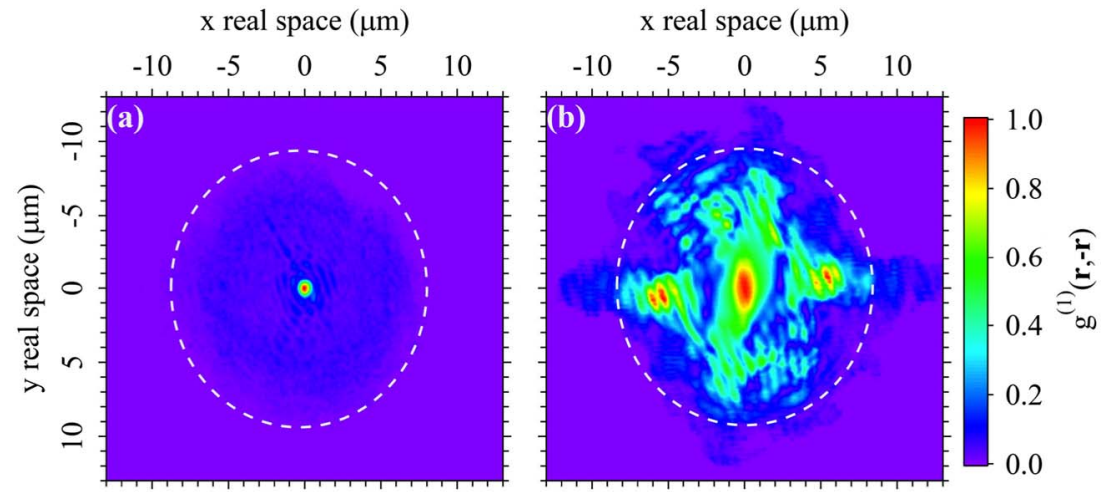

Fig. 2. (Color online) Spatial ordering of the polariton condensate. Below the threshold (0.3 Pth), the coherence is limited to the de Broglie wavelength of the polaritons. Above the threshold (1.9 Pth), significant correlations, disturbed by disorder, are observed up to the limits of the excitation spot.

a clear way to differentiate between the two effects? Indeed, the threshold behavior, the increase of the first-order coherence, the corresponding decrease in the linewidth, the changes in the second order coherence, and the appearance of spatial order are all expected for a VCSEL.

The first point to stress is that we are indeed dealing with polaritons. The first argument is that the blueshift of the lower polariton is much smaller than the Rabi splitting, the excitations of the system are indeed polaritons, and not photons on one side and excitons on the other. This is constantly monitored through the fact that we keep the upper branch of polaritons (see, for example, [62]). Both the upper and the lower branch appear at energies, which are clearly distinct from the original exciton or photon energies.

The CdTe sample that we have been studying also shows a VCSEL transition at much higher densities, a much better demonstration may be found in a paper by Bloch's group [줄, where they study in detail the differences between a polariton laser and a photon laser in a pillar microcavity, a device very close indeed to a VCSEL. As discussed earlier, the first threshold corresponds to the onset of stimulated scattering by lower polaritons. This threshold corresponds to a number of polaritons per state around 1. Above threshold, the emission line continues to blueshift because of polariton-polariton interactions. The second threshold, on the contrary, occurs in the weak coupling regime, where excitons are dissociated into electron-hole pairs. The threshold is then set by the Bernard Duraffourg condition [64], which is related to the Fermi distribution of electrons and holes and not to the Bose distribution of polaritons or photons. Then, the position of the laser line then corresponds precisely to the position of the photon cavity mode.

Going further, the phase that we and others observe possesses a number of well-defined features that make a clear difference with a VCSEL. The first one is the behavior of the second-order coherence. In a laser, and in particular in a VCSEL, the second-order coherence decreases from 2 to about 1 above threshold and tends to 1 , the limit value, as the power is increased above threshold [65]. In the case of a polariton condensate, the second-order coherence approaches 1 just above threshold, but g2 increases again at higher excitation density, as a result of the interactions between polaritons (see above) [45].

In a VCSEL, as in a polariton condensate, the spatial coherence is expected to increase in a major way when crossing the threshold. However, clear differences are expected, linked to the behavior below the threshold. In a polariton condensate, the coherence length is expected to be given by the de Broglie wavelength of polaritons when it should more or less correspond to the coherence length of excitons for the case of a VCSEL. It is clear that our experiments, which are not limited by our spatial resolution, give results corresponding quite accurately to the de Broglie wavelength of polaritons. It is safe then to state that, as far as we can infer, the phase observe just below threshold is indeed that of polaritons in quasi thermal equilibrium.

Importantly enough, few groups have now been able to observe clearly the two thresholds in the same microcavity sample at the same position. The first threshold is clearly observed in the strong coupling regime, and the second one in
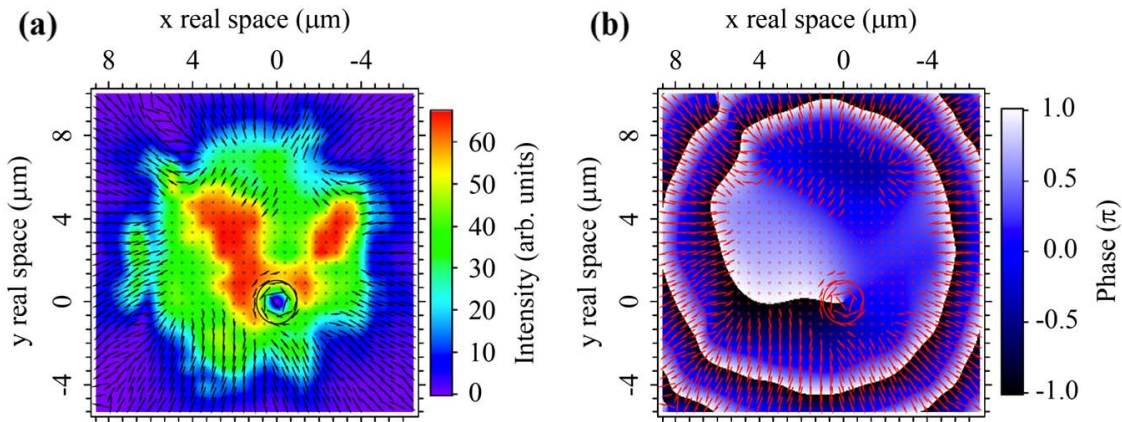

Fig. 3. (Color online) (a) Results of theoretical simulation showing the amplitude of the condensate in the presence of disorder. Black arrows measure the polariton flow that is obtained from the phase of the condensate shown in (b) as $\mathbf{k}=\nabla \varphi$ (reproduced from [56] with the agreement of the author). 
the weak-coupling regime. Bajoni et al. [6] observe the two thresholds in microcavity pillars, Snoke et al. [67] observe them in a stressed sample, and Azzini et al. [68] observe them in nanocavities.

It is central at this stage to correct a misuse of words: conventional lasing in semiconductors does not correspond to population inversion (which would mean having more electrons in the conduction band than electrons in the valence band). The lasing condition in semiconductors is the well-known Bernard-Durraffourg condition [63], i.e., that the distance between the quasi-Fermi levels for electron and holes is larger than the energy of the gap. It is therefore misleading to talk, in semiconductors, about lasing without inversion. What is important is that laser-like emission by the polariton condensate occurs orders of magnitude below the Bernard-Durraffourg density (similar to the Mott density) therefore reducing the power consumption of the device.

A number of groups have used the wording "polariton lasing" rather than "BEC of polaritons" (see, for example, Deng et al. [69] and Bajoni et al. [67]), the difference being understood by the writers is linked to the fact that the observed transition is not observed in full thermal equilibrium. This may be right, however, no real BEC will satisfy all the conditions set be the initial theoretical description. In particular here, we are very far from a standard thermal equilibrium. This is not merely because of the fact that the involved times are so short, but also because of the fact that the polariton quantum fluid is only a small portion of the total density at a given place in the cavity.

The first point may be quite easily overcome, as what matters is whether the polariton gas is able to get to thermal equilibrium within the average lifetime of polaritons. Different groups have been able to show that, in proper conditions, this was indeed the case. The second point is more difficult as the polariton quantum phase overlaps spatially with the exciton reservoir. The Bogoliubov equations that have been developed by our good friend theorists [70] allow to describe in detail such an interaction and we may consider that, as the interaction is in fact rather weak, the approximation of an almost isolated condensate is reasonable. A third issue also comes into play at this point : the fact that we are dealing with a two dimensional system. In such a case, for an infinite system, one does not expect BEC but rather a Berezinskii, Kosterlitz, Thouless transition [71]. This point has not been

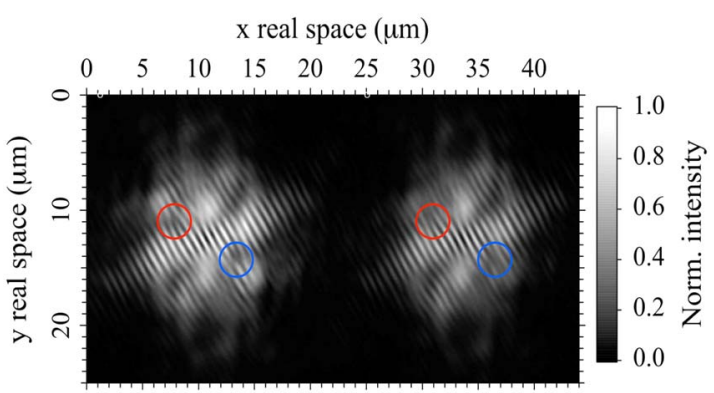

Fig. 4. (Color online) Interference fringes observed with the polarization resolved interferometer. On the left of the image appears the sigma 1 polarization and on the right, the sigma-. Dislocations forks are clearly observed (red and blue circles) in the left part of the image, but do not have a counterpart on the right side. These correspond to half-vortices. studied in detail yet as the size of the spot is difficult to modify at will.

It is now admitted that a coherent phase of polaritons, with the very interesting properties such as superfluidity that go with it, may be observed in at least two well-defined cases: spontaneous build-up upon nonresonant excitation or direct creation through resonant excitation.

\section{WHERE PHOTON LASING CANNOT EXPLAIN WHAT IS OBSERVED}

\section{A. Vortices}

Quantized vortices are one of the prominent signatures of superfluidity, they were initially assumed by Onsager, in a paper that is basically not cited [72]. Since then, a very large number of theoretical works as well as an equivalent quantity of experimental works have brought quantized vortices at the core of superfluid behavior. A proper choice of references is very difficult to make, with however seminal papers such as for example the work of Pitaevskii and coworkers on the theory side [73] or the works of Cornell et al. [74], Ketterle et al. [75], or the work of Dalibard et al. [76] as seminal examples on the experimental side.

It has often been opposed to our observation (see Fig. 4) the fact that vortices may also be observed in conventional lasers or VCSELs [56]. Amazingly enough, although this comment is perfectly right, the experimental observation of vortices in lasers is quite scarce. This might be due to the fact that the polarization of most lasers is set by the geometry of the laser, including the Brewster windows for the gas lasers, and that there is no degeneracy between cross-polarized states. Vortex lattices have been observed in very few experiments (see, for example, $[\underline{77,78]}]$ ) in conditions that clearly differ from what we are observing here. Vortices are now observed in many different configurations in polariton quantum fluids. The first observation is due to Konstantinos Lagoudakis and Augustin Baas in CdTe spontaneously formed vortices [79]. This observation has been followed by a succession of very interesting works performed either in the OPO configuration $[\underline{80,81]}$ or by directly driving the quantum fluid of polaritons, that show both static and dynamic vortices in polariton fluids, something that cannot have an equivalent in lasers.

\section{B. Half-Vortices}

Polaritons carry a spin, which originates both from their exciton and from their photon content. As a result, polariton condensates are spinor condensates. It has been predicted, in such a case, that novel vertical entities should be observed: half-vortices $[\underline{82}, \underline{83}, \underline{84}]$. Half-vortices correspond to a phase winding of $\pi$ accompanied by a spin flip of the bosonic particle. They have been clearly evidenced by Konstantinos Lagoudakis in the case of polariton condensates [85]. Halfvortices should also be observable in VCSELs as photons also carry spin. An extensive search did not allow us to find any experimental finding corresponding to the observation of halfvortices in lasers.

\section{Bogoliubov Dispersion}

Lasing in VCSELs corresponds to the interaction of an electron-hole plasma with the photon mode of the cavity. This photon mode shows a parabolic dispersion that is basically 
not modified across threshold. On the contrary, polariton condensates may be described, as all superfluids, through the Bogoliubov theory of weakly interacting Bose gases [86]. Within this theory, excitations of a superfluid, give rise to a linearization of the dispersion relation, that comes from the coupling of excitations with the counterpropagating modes, the so called "ghost branch." Direct observation of the ghost branch has been provided by a seminal work by Ketterle's group in the case of atom condensates [87]. The existence of the ghost branch has been very recently presented in the work of Konhle [88] and provides evidence that standard photon lasing cannot explain the observations in the case of polariton condensates.

\section{Josephson Junctions}

In 1964, Josephson pointed out the possibility of Josephson oscillations between two superconductors separated by a thin insulating layer [89]. This idea was at the same time mind shaking and demonstrated by experiments. It led to the wellknown field of Josephson oscillations, considered as a possible basis for quantum computing [90]. Josephson Junctions, initially considered for the case of two superconducting layers, are also expected to occur between two bosonic condensates separated by a barrier [91]. Thanks to the disorder that occurs in our CdTe microcavities, we have been able to observe Josephson oscillations between to localized disorder minima in our sample [92]. Such oscillations are really specific of two coupled BECs and cannot be explained by the temporal behavior of two coupled VCSELs. In such a case, the phase oscillations would be sinusoidal, as in the case of two coupled oscillators, and would not show a saw-tooth behavior as demonstrated in the experiment.

\section{E. 1D Condensates}

The Bloch's group has recently been able to prepare $1 \mathrm{D}$ microcavity samples with a quality that is sufficient to observe condensation under nonresonant excitation at a precise position along the length of the microcavity wire [93]. The behavior of this sample, of the highest quality by all standards, cannot be explained in any way when using a laser based approach. Coherent emission is indeed observed tens of microns away from the excitation spot.

\section{NEXT STEPS}

It is now admitted that a coherent phase of polaritons, with the very interesting properties such as superfluidity that go with it, may be observed in at least two well-defined cases. The first case, discussed at length in the present review, correspond to a spontaneous phase transition, with all limits with respect to the "ideal case" of $3 \mathrm{D}$, infinite in dimensions and in lifetime, no interaction BEC. We understood over the last years that such ideal conditions were basically never present, not preventing us in any way to observe the marvelous properties of the Bose condensates.

The second case that is now proving to be of major interest in many different respects, is the case where a quantum fluid is created trough direct excitation, may it be TOPO processes or even direct excitation into the polariton branch. There again, the magical properties of superfluids are obtained, with quasiparticles that possess a mass four or five orders of magnitude less than electrons. We then have at hand a quantum fluid that we can manipulate at will. This field is clearly very promising. A dream come true for all solid state physicists!

\section{ACKNOWLEDGMENTS}

This work is inspired from the successive PhD works in my group (but not only as I integrate in this review the works of Senellart, Richard, Kasprzak, and others) over the past 15 years, starting from the PhD of Saba, when we first envisaged the possibility of condensation of polaritons and designed an original way to produce efficient trapping of polaritons. This work has been followed by what I call the dream team of LOEQ with, in order of appearance on stage, Kundermann, Kasprzak, Kaitouni, El Daïf, Lagoudakis, Paraïso, Nardin, Cerna, Kohnle, Manni, and Grosso. It has has also been supported very strongly by a group of extremely talented postdocs, which I wish to congratulate warmly for their outstanding work: Richard, Baas, Guillet, Pietka and, last but not least, Léger. I also would like to convey my most sincere thanks to the theoreticians who allowed us to understand our results, by spending enough time with us and by putting into simple words the results of their equations. In particular, M. Wouters, V. Savona, C. Ciuti, A. Kavokin, and Y Rubo deserve very special acknowledgements. A. Quattropani and P. Schwendiman have also helped with many discussions and their indefectible support of theorist-experimentalist collaborations. Last, but not least, this work would not have been possible without the very close collaboration with Dang's group in Grenoble, and in particular without the very high quality samples prepared by R. André. The work has been carried out within the framework of the Quantum Photonics National Center of Competence in research financed by the Swiss National Science Foundation. Complementary funding for conferences and meetings has been obtained through the Latsis Foundation, the Polatom network of the European Science Foundation, and a King Saud University associate professorship.

\section{REFERENCES}

1. N. Bose, "Plancks Gesetz und Lichtquantenhypothese," Z. Phys. D 26, 178-181 (1924).

2. A. Einstein, "Quantentheorie des einatomigen idealen gases I," Sitzungsber. Kgl. Preuss. Akad. Wiss., 1, 3-14 (1925).

3. H. K. Onnes, "Further experiments with liquid helium. D. On the change of electric resistance of pure metals at very low temperatures, etc. V. The disappearance of the resistance of mercury," Commun. Phys. Lab. Univ. Leiden 122b (1911).

4. J. Bardeen, L. N. Cooper, and J. R. Schrieffer, "Theory of superconductivity," Phys. Rev. 108, 1175-1204 (1957).

5. P. Kapitza, "Viscosity of liquid helium below the $\lambda$-point," Nature 141, 74-75 (1938).

6. J. F. Allen and A. D. Misener, "Flow phenomena in liquid helium II," Nature 142, 643-644 (1938).

7. F. London, "The $\lambda$-phenomenon of liquid helium and the BoseEinstein degeneracy" Nature, 141, 643-644 (1938).

8. L. D. Landau, "Theory of the superfluidity of Helium II," Phys. Rev. 60, 356-358 (1941).

9. N. N. Bogoliubov, "On the theory of superfluidity," J. Phys. USSR 11, 23-32 (1947).

10. S. A. Moskalenko, "Reversible optico-hydrodynamic phenomena in a nonideal. exciton gas," Solid State Commun. 4, 199-204 (1962).

11. J. M. Blatt, K. W. Böer, and W. Brandt, "Bose-Einstein condensation of excitons," Phys. Rev. 126, 1691-1692 (1962).

12. L. V. Keldysh and A. N. Kozlov, "Collective properties of excitons on semiconductors," Sov. Phys. JETP 27, 521-528 (1968). 
13. L. L. Chase, N. Peyghambarian, G. Grynberg, and A. Mysyrowicz, "Evidence for Bose-Einstein condensation of biexcitons in CuCl," Phys. Rev. Lett. 42, 1231-1234 (1979).

14. D. Hulin, A. Mysyrowicz, and C. Benoit àl a Guillaume, "Evidence for Bose-Einstein statistics of an exciton gas," Phys. Rev. Lett. 45, 1970-1973 (1980).

15. J. A. Kash, M. Zachau, E. E. Mendez, and J. M. Hong, "FermiDirac distribution of excitons in coupled quantum wells," Phys. Rev. Lett. 66, 2247-2250 (1991).

16. L. V. Butov and A. I. Filin, "Anomalous transport and luminescence of indirect excitons in AlAs/GaAs coupled quantum wells as evidence for exciton condensation," Phys. Rev. B58, 19802000 (1998).

17. L. V. Butov, C. W. Lai, A. L. Ivanov, A. C. Gossard, and D. S. Chemla, "Towards Bose-Einstein condensation of excitons in potential traps," Nature 417, 47-52 (2002).

18. L. V. Butov, A. C. Gossard, and D. S. Chemla, "Macroscopically ordered state in an exciton system," Nature 418, 751-754 (2002)

19. S. A. Moskalenko and D. W. Snoke, eds. Bose-Einstein Condensation of Excitons and Biexcitons and Coherent Nonlinear Optics with Excitons (Cambridge University, 2000).

20. N. F. Mott, Metal Insulator Transitions, 2nd ed. (Taylor \& Francis, 1990).

21. L. Kappei, J. Szczytko, F. Morier-Genoud, and B. Deveaud, "Direct observation of the Mott transition in an optically excited semiconductor quantum well," Phys. Rev. Lett. 94, 147403 (2005).

22. M. Stern, V. Garmider, V. Umansky, and I. Bar-Joseph, "Mott transition of excitons in coupled quantum wells," Phys. Rev. Lett. 100, 256402 (2008).

23. D. Semkat, W.-D. Kraeft, G. Manzke, D. Krempe, and K. Henneberger, "Ionization equilibrium in an excited semiconductor: Mott transition versus Bose-Einstein condensation," Phys. Rev. B81, 155201 (2009).

24. V. V. Nikolaev and M. E. Portnoi, "Theory of excitonic Mott transition in double quantum wells," Phys. Status Solidi C 1, 1357-1362 (2004).

25. D. Snoke, "Predicting the ionization threshold for carriers in excited semiconductors," Solid State Commun. 146, 73-77 (2008).

26. J. J. Hopfield, "Theory of the contribution of excitons to the complex dielectric constant of crystals," Phys. Rev. 112, 1555-1567 (1958).

27. P. D. Lett, R. N. Watts, C I. Westbrook, W. D. Phillips, P. L. Gould, and H. J. Metcalf, "Observation of atoms laser cooled below the Doppler limit," Phys. Rev. Lett. 61, 169-172 (1988).

28. M. H. Anderson, J. R. Ensher, M. R. Matthews, C. E. Wieman, and E. A. Cornell, "Observation of Bose-Einstein condensation in a dilute atomic vapor," Science 269, 198-201 (1995).

29. K. B. Davis, M. O. Mewes, M. R. Andrews, N. J. van Druten, D. S. Durfee, D. M. Kurn, and W. Ketterle, "Bose-Einstein condensation in a gas of sodium atoms," Phys. Rev. Lett. 75, 3969-3973 (1995).

30. D. S. Hall, M. R. Matthews, C. E. Wieman, and E. A. Cornell, "Measurements of relative phase in two-component BoseEinstein condensates," Phys. Rev. Lett. 81, 1543-1546 (1998).

31. M. R. Andrews, C. G. Townsend, H.-J. Miesner, D. S. Durfee, D. M. Kurn, and W. Ketterle, "Observation of interference between two Bose condensates," Science 275, 637-641 (1997).

32. J. R. Abo-Shaeer, C. Raman, J. M. Vogels, and W. Ketterle, "Observation of vortex lattices in Bose-Einstein condensates," Science 292, 476-479 (2001).

33. C. Weisbuch, N. Nishioka, A. Ishikawa, and Y. Arakawa, "Observation of the coupled exciton-photon mode splitting in a semiconductor quantum microcavity," Phys. Rev. Lett. 69, 3314-3317 (1992).

34. L. Pitaevskii and S. Stringari, Bose-Einstein Condensation (Clarendon, 2003).

35. A. Imamoglu, R. J. Ram, S. Pau, and Y. Yamamoto, "Nonequilibrium condensates and lasers without inversion: excitonpolariton lasers," Phys. Rev. A53, 4250-4253 (1996).

36. R. Houdre, J. L. Gibernon, P. Pellandini, R. P. Stanley, U. Oesterle, C. Weisbuch, J. O'Gorman, B. Roycroft, and M. Ilegems, "Saturation of the strong-coupling regime in a semiconductor microcavity: free-carrier bleaching of cavity polaritons," Phys. Rev. B 52, 7810-7813 (1995)
37. P. G. Savvidis, J. J. Baumberg, R. M. Stevenson, M. S. Skolnick, D. M. Whittaker, and J. S. Roberts, "Angle-resonant stimulated polariton amplifier," Phys. Rev. Lett. 84, 1547-1550 (2000).

38. M. Saba, C. Ciuti, J. Bloch, V. Thierry-Mieg, R. André, L. S. Dang, S. Kundermann, A. Mura, G. Bongiovanni, J. L. Staehli, and B. Deveaud, "High-temperature ultrafast polariton parametric amplification in semiconductor microcavities," Nature, $\mathbf{4 1 4}$ 731-735 (2001)

39. G. Malpuech, A. Di Carlo, A. Kavokin, J. J. Baumberg, A. Zamfirescu, and P. Lugli, "Room-temperature polariton lasers based on GaNmicrocavities," Appl. Phys. Lett. 81, 412-414(2002).

40. J. J. Baumberg, A. V. Kavokin, S. Christopoulos, A. J. D. Grundy, R. Butté, G. Christmann, D. D. Solnyshkov, G. Malpuech, G. B. H. von Högersthal, E. Feltin, J.-F. Carlin, and N. Grandjean, "Spontaneous polarization buildup in a room-temperature polariton laser," Phys. Rev. Lett. 101, 136409 (2008).

41. O. Penrose and L. Onsager, "Bose-Einstein condensation and liquid helium," Phys. Rev. 104, 576-584 (1956)

42. L. S. Dang, H. Heger, R. André, F. Boeuf, and R. Romestain, "Stimulation of polariton photoluminescence in semiconductor microcavity," Phys. Rev. Lett. 81, 3920-3923 (1998)

43. P. Senellart and J. Bloch, "Nonlinear emission of microcavity polaritons in the low density regime," Phys. Rev. Lett. 12331236 (1999)

44. J. Kasprzak, "Condensation of exciton polaritons," $\mathrm{PhD}$ thesis (Université Joseph Fourier-Genoble 1, 2006).

45. A. Amo, D. Sanvitto, F. P. Laussi, D. Ballarini, E. del Valle, M. D Martin, A. Lemaître, J. Bloch, D. N. Krizhanovskii, M. S. Skolnick, C. Tejedor, and L. Viña, "Collective fluid dynamics of a polariton condensate in a semiconductor microcavity," Nature 457, 291295 (2009).

46. M. Richard,"Quasi-condensation de polaritons sous excitation incoherente dans les microcavities II-VI a base de CdTe," $\mathrm{PhD}$ thesis (Université Joseph Fourier-Genoble 1, 2004).

47. R. M. Stevenson, V. N. Astratov, M. S. Skolnick, D. M. Whittaker, M. Emam-Ismail, A. I. Tartakovskii, P. G. Savvidis, J. J. Baumberg, and J. S. Roberts, "Continuous wave observation of massive polariton redistribution by stimulated scattering in semiconductor microcavities," Phys. Rev. Lett. 85, 3680-3683 (2000).

48. P. Senellart, J. Bloch, B. Sermage, and J. Y. Marzin, "Microcavity polariton depopulation as evidence for stimulated scattering," Phys. Rev. B63, R16263-16266 (2000).

49. D. N. Krizhanovskii, K. G. Lagoudakis, M. Wouters, B. Pietka, R. A. Bradley, K. Guda, D. M. Whittaker, M. S. Skolnick, B. Deveaud-Plédran, M. Richard, R. André, and Le Si Dang, "Coexisting nonequilibrium condensates with long-range spatial coherence in semiconductor microcavities," Phys. Rev. B80 045317 (2008)

50. H. Deng, G. Weihs, C. Santori, J. Bloch, and Y. Yamamoto, "Condensation of semiconductor microcavity exciton polaritons," Science 298, 199-202 (2002).

51. N. G. Vy, H. T. Cao, D. B. Tran Thoai, and H. Haug, "Time dependence of the ground-state population statistics of condensed microcavity polaritons," Phys. Rev. B80, 195306 (2009).

52. J. Kasprzak, M. Richard, A. Baas, B. Deveaud, R. Andre, J.-Ph. Poizat, and L. S. Dang, "Second-order time correlations within a polariton Bose-Einstein condensate in a CdTe microcavity," Phys. Rev. Lett. 100, 067402 (2008).

53. M. Aßmann, J.-S. Tempel, F. Veit, M. Bayer, A. Rahimi-Iman, A Löffler, S. Höfling, S. Reitzenstein, L. Worschech, and A. Forchel, "From polariton condensates to highly photonic quantum degenerate states of bosonic matter," Proc. Natl. Acad. Sci. USA 108, 1804-1809 (2011).

54. P. Schwendimann and A. Quattropani, "Statistics of the polariton condensate," Phys. Rev. 77, 085317 (2008).

55. J. Kasprzak, M. Richard, S. Kundermann, A. Baas, P. Jeambrun, J. M. J. Keeling, F. M. Marchetti, M. H. Szymańska, R. André, J. L. Staehli, V. Savona, P. B. Littlewood, B. Deveaud, and Le Si Dang, "Bose-Einstein condensation of exciton polaritons," Nature 443, 409-414 (2006).

56. J. Scheuer and M. Orenstein, "Optical vortices crystals: spontaneous generation in nonlinear semiconductor microcavities," Science 285, 230-233 (1999).

57. R. Ballili, V. Hartwell, D. Snoke, L. Pfeiffer, and K. West, "BoseEinstein condensation of microcavity polaritons in a trap," Science 316, 1007-1010 (2007). 
58. H. Deng, D. Press, S. Götzinger, G. S. Solomon, R. Hey, K. H. Ploog, and Y. Yamamoto, "Spatial coherence of a polariton condensate," Phys. Rev. Lett. 99, 126403 (2007).

59. E. Wertz, L. Ferrier, D. D. Solnyshkov, R. Johne, D. Sanvitto, A. Lemaître, I. Sagnes, R. Grousson, A. V. Kavokin, P. Senellart, G. Malpuech, and J. Bloch, "Spontaneous formation and optical manipulation of extended polariton condensates," Nat. Phys. 6, 860-864 (2010).

60. S. Christopoulos, G. Baldassarri Höger von Högersthal, A. J. D. Grundy, P. G. Lagoudakis, A. V. Kavokin, J. J. Baumberg, G. Christmann, R. Butté, E. Feltin, J.-F. Carlin, and N. Grandjean, "Room-temperature polariton lasing in semiconductor microcavities," Phys. Rev. Lett. 98, 126405 (2007).

61. G. Christmann, R. Butté, E. Feltin, J.-F. Carlin, and N. Grandjean, "Room temperature polariton lasing in a GaN/AlGaN multiple quantum well microcavity," Appl. Phys. Lett. 93, 051102 (2008).

62. E. Wertz, L. Ferrier, D. D. Solnyshkov, P. Senellart, D. Bajoni, A. Miard, A. Lemaître, G. Malpuech, and J. Bloch, "Spontaneous formation of a polariton condensate in a planar GaAs microcavity," Appl. Phys. Lett. 95, 051108 (2009).

63. D. Bajoni, P. Senellart, A. Lemaître, and J. Bloch, "Photon lasing in GaAs microcavity: similarities with a polariton condensate," Phys. Rev. B76, 201305 (2007)

64. M. G. A. Bernard and G. Duraffourg, "Laser conditions in semiconductors," Phys. Status Solidi 1, 699-703 (1961).

65. S. M. Ulrich, C. Gies, S. Ates, J. Wiersig, S. Reitzenstein, C. Hofmann, A. Löffler, A. Forchel, F. Jahnke, and P. Michler, "Photon statistics of semiconductor microcavity lasers," Phys. Rev. Lett. 98, 043906 (2007).

66. D. Bajoni, P. Senellart, E. Wertz, I. Sagnes, A. Miard, A. Lemaître, and J. Bloch, "Polariton laser using single micropillar GaAsGaAlAs semiconductor cavities," Phys. Rev. Lett. 100, 047401 (2008).

67. B. Nelsen, R. Balili, D. W. Snoke, L. Pfeiffer, and K. West, "Lasing and polariton condensation: two distinct transitions in GaAs microcavities with stress traps,” J. Appl. Phys. 105, 122414 (2009).

68. S. Azzini, D. Gerace, M. Galli, I. Sagnes, R. Braive, A. Lemaître, J. Bloch, and D. Bajoni, "Ultra-low threshold polariton lasing in photonic crystal cavities," Appl. Phys. Lett. 99, 111106 (2011).

69. H. Deng, G. Weihs, D. Snoke, J. Bloch, and Y. Yamamoto, "Polariton lasing vs. photon lasing in a semiconductor microcavity," Proc. Natl. Acad. Sci. USA 100, 15318-15323 (2003).

70. D. Sarchi, M. Wouters, and V. Savona, "Polariton parametric photoluminescence in spatially inhomogeneous systems," Phys. Rev., B79, 165315 (2009).

71. Z. Hadzibabic, P. Krüger, M. Cheneau, B. Battelier, andJ. Dalibard, "Berezinskii-Kosterlitz-Thouless crossover in a trapped atomic gas," Nature 441, 1118-1121 (2006).

72. L. Onsager, "Statistical hydrodynamics," Il Nuevo Cimento 6, 279-287 (1949).

73. F. Dalfovo, S. Giorgini, L. Pitaevskii, and S. Stringari, "Theory of Bose-Einstein condensation in trapped gases," Rev. Mod. Phys. 71, 463-512 (1999)

74. M. R. Matthews, B. P. Anderson, P. C. Haljan, D. S. Hall, C. E. Wiemann, and E. A. Cornell, "Vortices in a Bose-Einstein condensate," Phys. Rev. Lett. 83, 2498-2501 (1999).

75. J. R. Abo-Shaeer, C. Raman, J. M. Vogels, and W. Ketterle, "Observation of vortex lattices in Bose-Einstein condensates," Science 292, 476-479 (2001).
76. K. W. Madison, F. Chevy, W. Wohlleben, and J. Dalibard, "Vortex formation in a stirred Bose-Einstein condensate," Phys. Rev. Lett. 84, 806-809 (2000).

77. Y. F. Chen and Y. P. Lan, "Formation of optical vortex lattices in solid-state microchip lasers: spontaneous transverse mode locking," Phys. Rev. A64, 063807 (2001).

78. Y. F. Chen, K. F. Huang, H. C. Lai, and Y. P. Lan, "Observation of vector vortex lattices in polarization states of an isotropic microcavity laser," Phys. Rev. Lett. 90, 053904 (2003).

79. K. Lagoudakis, M. Wouters, M. Richard, A. Baas, I. Carusotto, R. André, L. S. Dang, and B. Deveaud-Plédran, "Quantized vortices in an exciton-polariton condensate," Nat. Phys. 4, 706-710 (2008)

80. A. Amo, J. Lefrére, S. Pigeon, C. Adrados, C. Ciuti, I. Carusotto, R. Houdré, E. Giacobino, and A. Bramati, "Superfluidity of polaritons in semiconductor microcavities," Nature 457, 291-295 (2009).

81. D. Sanvitto, S. Pigeon, A. Amo, D. Ballarini, M. De Giorgi, I Carusotto, R. Hivet, F. Pisanello, V. G. Sala, P. S. S. Guimaraes, R. Houdré, E. Giacobino, C. Ciuti, A. Bramati, and G. Gigli, "Persistent currents and quantized vortices in a polariton superfluid," Nat. Phys. 6, 527-533 (2010).

82. G.E. Volovik and V. P. Mineev, "Hydrodynamics of the A phase of superfluid ${ }^{3} \mathrm{He}$," Sov. Phys. JETP 44, 591-599 (1976).

83. W. F. Brinkman, "Textural singularities in the superfluid A phase of 3He," J. Low Temp. Phys. B 26, 165-191 (1977).

84. Y. G. Rubo, "Half vortices in exciton polariton condensates," Phys. Rev. Lett. 99, 106401 (2007).

85. K. Lagoudakis, T. Ostatnick, A. V. Kavokin, Y. G. Rubo, R. André, and B. Deveaud-Plédran, "Observation of half-quantum vortices in an exciton-polariton condensate," Science 326, 974-976 (2009)

86. N. N. Bogoliubov, "On a new method in the theory of superconductivity," Il Nuevo Cimento 7, 794-805 (1958).

87. J. M. Vogels, K. Xu, C. Raman, J. R. Abo-Shaeer, and W. Ketterle, "Experimental observation of the Bogoliubov transformation for a Bose-Einstein condensed gas," Phys. Rev. Lett. 88, 060402 (2002).

88. V. Kohnle, Y. Léger, M. Wouters, M. Richard, M. T. PortelaOberli, and B. Deveaud-Plédran, "From single particle to superfluid excitations in a dissipative polariton gas," Phys. Rev. Lett 106, 255302 (2011).

89. B. D. Josephson, "Coupled superconductors," Rev. Mod. Phys. 36, 216-220 (1964).

90. Y. Mahklin, G. Schön, and A. Schnirman, "Quantum-state engineering with Josephson-junction devices," Rev. Mod. Phys. 73, 357-400 (2001)

91. S. Raghavan, A. Smerzi, S. Fantoni, and S. R. Shenoy, "Coherent oscillations between two weakly coupled Bose-Einstein condensates: Josephson effects, $\pi$ oscillations, and macroscopic quantum self-trapping," Phys. Rev., A59, 620-633 (1999).

92. K. Lagoudakis, B. Pietka, M. Wouters, R. André, and B. DeveaudPlédran, "Coherent oscillations in an exciton-polariton Josephson junction,” Phys. Rev. Lett. 105, 120403 (2010).

93. E. Wertz, L. Ferrier, D. D. Solnyshkov, R. Johne, D. Sanvitto, A. Lemaître, I. Sagnes, R. Grousson, A. V. Kavokin, P. Senellart, G. Malpuech, and J. Bloch, "Spontaneous formation and optical manipulation of extended polariton condensates," Nat. Phys. 6, 860-864 (2010). 